\title{
Resolution in focused electron- and ion-beam induced processing
}

\author{
Ivo Utke ${ }^{\mathrm{a})}$ \\ EMPA, Swiss Federal Laboratories for Materials Testing and Research, Laboratory for Mechanics \\ of Materials and Nanostructures, Feuerwerkerstr. 39, CH-3602 Thun, Switzerland \\ Vinzenz Friedli \\ EMPA, Swiss Federal Laboratories for Materials Testing and Research, Laboratory for Mechanics \\ of Materials and Nanostructures, Feuerwerkerstr. 39, CH-3602 Thun, Switzerland \\ and Advanced Photonics Laboratory, Ecole Polytechnique Fédérale de Lausanne (EPFL), CH-1015 \\ Lausanne, Switzerland \\ Martin Purrucker and Johann Michler \\ EMPA, Swiss Federal Laboratories for Materials Testing and Research, Laboratory for Mechanics \\ of Materials and Nanostructures, Feuerwerkerstr. 39, CH-3602 Thun, Switzerland
}

(Received 8 June 2007; accepted 27 August 2007; published 7 December 2007)

\begin{abstract}
The key physical processes governing resolution of gas-assisted focused electron-beam and ion-beam induced deposition and etching are analyzed via an adsorption rate model. The authors quantify how the balance of molecule depletion and replenishment determines the resolution inside the locally irradiated area. Scaling laws are derived relating the resolution of the deposits to molecule dissociation, surface diffusion, adsorption, and desorption. Supporting results from deposition experiments with a copper metalorganic precursor gas on a silicon substrate are presented and discussed. ( 2007 American Vacuum Society. [DOI: 10.1116/1.2789441]
\end{abstract}

\section{INTRODUCTION}

Local electron-beam induced chemical vapor deposition (CVD) is a physical phenomenon well known from the buildup of carbon contamination since the beginning of electron microscopy. ${ }^{1}$ Recent research has spent tremendous efforts on the systematic creation of functional nanoscale deposits by means of focused electron beams and-with the development of scanning ion microscopes - of focused ion beams. Organic, organometallic, and inorganic precursor gas molecules were supplied into the microscope chamber. Upon irradiation, deposition results from nonvolatile dissociation products, whereas etching occurs when a reaction of dissociation products with the substrate leads to the formation of volatile species. These local deposition and etching techniques have numerous potential applications in nanosciences including fabrication of attachments in mechanics, ${ }^{2}$ highresolution sensors in magnetic, thermal, and optical scanning probe microscopy, ${ }^{3-5}$ optical elements in nanooptics, ${ }^{6,7}$ contacts in electronics, ${ }^{8}$ and nanopores for ionic current measurements of cells and DNA in biology. ${ }^{9,10}$

Few experiments analyze the physics of focused electronbeam (FEB) and focused ion-beam (FIB) induced deposition and etching. For FEB, Allen et al. ${ }^{11}$ noted that the spatial flux distribution of electrons passing through the adsorbed molecule layer consists of the incident primary beam (of up to several $\mathrm{keV}$ ) and the emitted electrons: backscattered primaries and low-energy $(\leq 50 \mathrm{eV})$ secondary electrons. The entire energy spectrum is responsible for the dissociation of surface adsorbed molecules. For FIB, Dubner et al. ${ }^{12}$ pro-

$\overline{{ }^{a}}$ Electronic mail: ivo.utke@empa.ch posed that this spectrum is associated to the energy deposited into the substrate surface through the collision cascade generated by the primary ion beam.

Our work is motivated by the fact that focused particle beam induced chemical vapor deposition and etching processes are widely used in nanoscale fabrication, but there are only very few attempts to describe the spatial resolution of this process theoretically. For a singular primary electron beam, Silvis-Cividjian et al. ${ }^{13}$ and Hagen et al. ${ }^{14}$ concluded from Monte Carlo simulations that the ultimate resolution depends on the emitted secondary electron distribution. However, their assumption that the irradiated area is permanently covered with a monolayer of adsorbed molecules is idealized. In fact, as we will show, this coverage results from a balance of molecule depletion by dissociation and molecule replenishment strongly depending on adsorption, desorption, and diffusion. Müller's model ${ }^{15}$ for FEB induced deposition, which was later adapted by Haraichi et al. ${ }^{16}$ to gas-assisted ion-beam induced etching, takes the key processes of surface diffusion, dissociation, desorption, and adsorption into account via an adsorption rate equation. However, he used a flat-top beam distribution which allows no conclusions about resolution.

In this article we present an adsorption rate model considering two relevant peak distributions for the incident beam and for the emitted spectrum, and a nondissociative Langmuir adsorption term. It allows derivation of three scaling laws for resolution and estimation of important physical parameters of the process. Finally, we discuss results of carefully designed experiments, clearly supporting the theoretical conclusions. 
TABLE I. Typical ranges of incident peak flux $f_{0}$ and size $\mathrm{FWHM}_{B}$ of a focused electron beam $(5 \mathrm{keV}$, field emission) and an ion beam $\left(30 \mathrm{kV}, \mathrm{Ga}^{+}\right)$. Representative ranges for $\sigma$ were collected from Refs. 17-19 for FEB and from Refs. 20-23 for FIB

\begin{tabular}{ccccc}
\hline \hline & $\begin{array}{c}f_{0} \\
{\left[1 / \mathrm{nm}^{2} \mathrm{~s}\right]}\end{array}$ & $\begin{array}{c}\mathrm{FWHM}_{B} \\
{[\mathrm{~nm}]}\end{array}$ & $\begin{array}{c}\sigma \\
{\left[\mathrm{nm}^{2}\right]}\end{array}$ & $\begin{array}{c}\sigma f_{0} \\
{[1 / \mathrm{s}]}\end{array}$ \\
\hline FEB & $8 \times 10^{6}-5 \times 10^{7}$ & $2.5-100$ & $2 \times 10^{-4}-2 \times 10^{-1}$ & $2 \times 10^{3}-1 \times 10^{7}$ \\
FIB & $2 \times 10^{5}-5 \times 10^{6}$ & $7-100$ & $10-50$ & $2 \times 10^{6}-2.5 \times 10^{8}$ \\
\hline \hline
\end{tabular}

\section{THEORY}

The model assumes second-order kinetics of molecule dissociation by electrons. In a system with rotational symmetry the vertical FEB deposition or etch rate $R(r)$ (in units of dimension per unit time) as function of the distance $r$ from the center of the primary electron (PE) beam is thus ${ }^{11}$

$$
R(r)=V n(r) \int_{0}^{E_{\mathrm{PE}}} \sigma(E) f(E, r) d E \cong V n(r) \sigma f(r),
$$

where $V$ is the volume of the decomposed molecule or etched atom, $n(r)$ is the number of adsorbed molecules per surface unit, $\sigma(E)$ is the energy-dependent electron impact dissociation cross section, $E_{\mathrm{PE}}$ is the energy of the PEs, and $f(E, r)$ describes the spatial flux distribution of the electron energy spectrum generated by the PEs. Since the energy integral can be solved only approximatively due to missing $\sigma(E)$ data of adsorbed molecules and uncertain parameter estimates entering in the Monte Carlo simulation of the emitted electron distribution $f(E, r),{ }^{11}$ we use the simplified expression in Eq. (1), where $\sigma$ represents an integrated value over the energy spectrum. Such cross sections were measured for several relevant molecules. ${ }^{17-23}$ The spatial distribution $f(r)$ is a convolution of the Gaussian incident beam distribution $f(r) \propto \exp \left(-r^{2}\right)$ with a peak function for the emitted spectrum which can be roughly approximated by $f(r)$ $\propto \exp (-r)$. Full widths at half maximum (FWHM) of the emitted distributions range between $\sim 0.1 \mathrm{~nm}(200 \mathrm{keV})^{14}$ and $2 \mathrm{~nm}(1 \mathrm{keV}) .^{11}$ Similar considerations apply for FIB where the spatial distribution responsible for molecule dissociation is a convolution of the primary beam distribution with the distribution of excited surface atoms generated by the collision cascade. ${ }^{12}$ Its range can be roughly estimated to $2 \mathrm{~nm}$ for $50 \mathrm{keV} \mathrm{Kr}^{+}$ions using an approach and data in Ref. 24. From Table I, summarizing typical FWHM ranges and peak flux values $f_{0}$ of primary beams, it can be seen that in most cases the incident beam dominates the FWHM of the distribution of emitted secondary electrons or excited surface atoms. Thus, we will discuss the scaling laws for the Gaussian distribution rather than concentrate on the convolution aspect of $f(r)$.

Four key processes as depicted in Fig. 1 are considered to determine the surface density $n(r)$ of adsorbed molecules: (a) adsorption from the gas phase governed by the precursor flux $J$, the sticking probability $s$, and coverage $n / n_{0}$; (b) surface diffusion from the surrounding area to the irradiated area governed by the diffusion coefficient $D$ and the concentration gradient; (c) desorption of physisorbed molecules after a residence time $\tau$, (d) molecule dissociation governed by the product $\sigma f(r)$. For the molecule adsorption rate $d n / d t$ follows:

$$
\begin{aligned}
\frac{\partial n}{\partial t}= & \underbrace{s J\left(1-\frac{n}{n_{0}}\right)}_{\text {Adsorption }}+\underbrace{D\left(\frac{\partial^{2} n}{\partial r^{2}}+\frac{1}{r} \frac{\partial n}{\partial r}\right)}_{\text {Ditusion }} \\
& -\underbrace{\frac{n}{\tau}}_{\text {Desorption }}-\underbrace{\sigma f n}_{\text {Decomposition }} .
\end{aligned}
$$

The adsorption term in Eq. (2) describes a nondissociative Langmuir adsorption, where $n_{0}$ is the maximum monolayer density given by the inverse of the molecule size. This adsorption type accounts for surface sites already occupied by nondissociated precursor molecules and limits the coverage to $n_{0}$. All parameters other than $n=n(r, t)$ and $f=f(r, t)$ are considered constant.

Solving Eq. (2) for steady-state $(d n / d t=0)$ and neglecting the diffusion term, we obtain $n(r)=s J \tau_{\text {eff }}(r)$ with the effective residence time of the molecules $\tau_{\text {eff }}(r)=\left(s J / n_{0}+1 / \tau\right.$ $+\sigma f(r))^{-1}$. The deposition or etch rate becomes

$$
R(r)=s J \tau_{\text {eff }}(r) \operatorname{V\sigma f}(r),
$$

and represents the deposit or etch shape at a given time. For any peak function $f(r)$ with a peak value $f_{0}=f(r=0)$, we can define the effective residence time in the center of the elec-

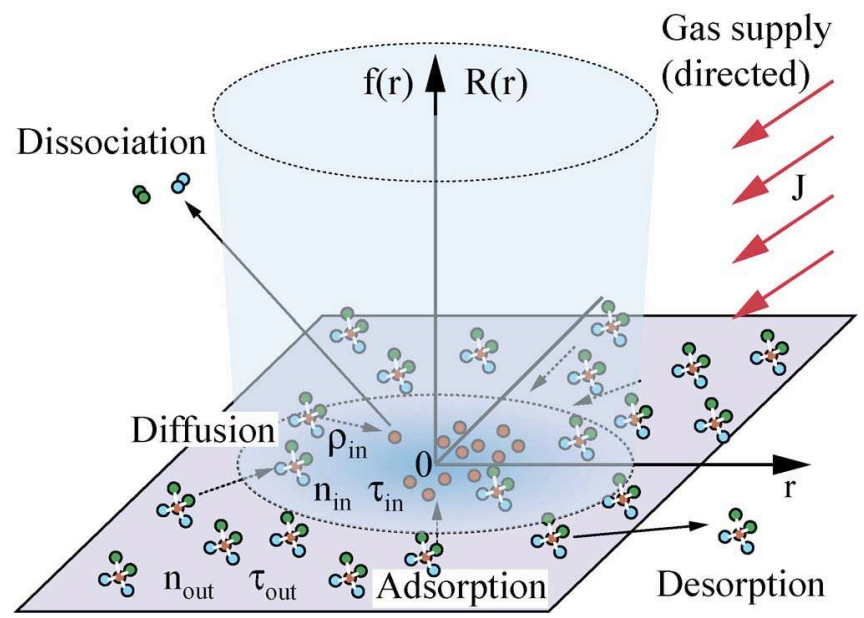

FIG. 1. Reference system and schematics of processes involved in FEBinduced deposition. Inside the irradiated area precursor molecules are depleted by dissociation and replenished by adsorption and surface diffusion (dashed arrows). Symbols are defined in the text. 
tron beam $\tau_{\text {in }}=\tau_{\text {eff }}(0)=1 /\left(s J / n_{0}+1 / \tau+\sigma f_{0}\right)$ and the effective residence time far away from the electron beam center $\tau_{\text {out }}$ $=\tau_{\text {eff }}(r \rightarrow \infty)=1 /\left(s J / n_{0}+1 / \tau\right)$. The dimensionless ratio $\tilde{\tau}$ $=\tau_{\text {out }} / \tau_{\text {in }}=1+\sigma f_{0} /\left(1 / \tau+s J / n_{0}\right)$ represents a measure for depletion of precursor molecules due to dissociation at the center of the beam. Furthermore, we define the dimensionless deposit or etch resolution as $\widetilde{\varphi}=\mathrm{FWHM}_{D} / \mathrm{FWHM}_{B}$, where $\mathrm{FWHM}_{D}$ and $\mathrm{FWHM}_{B}$ are the full widths at half maximum of $R(r)$, i.e. of the deposit or etch hole, and $f(r)$, respectively. With a Gaussian beam $f(r)=f_{0} \exp \left(-r^{2} / 2 a^{2}\right)$, we derive the first scaling law of resolution as a function of depletion $\widetilde{\varphi}(\widetilde{\tau})$,

$$
\widetilde{\varphi}=\left\{\log _{2}(1+\widetilde{\tau})\right\}^{1 / 2} .
$$

The idealized case of zero depletion, i.e., permanent monolayer coverage, corresponds to $\tilde{\tau}=1$. Then, deposition or etching proceeds in the electron-limited regime and the deposit (or etch) size corresponds to the electron beam distribution since the logarithmic term becomes 1 . With increasing depletion the deposit (or etch) size becomes steadily larger than the beam size. The degree of depletion strongly depends on the dissociation frequencies $\sigma f_{0}$ summarized for FEB and FIB in Table I. Reported desorption frequencies are situated around $\tau^{-1}=10^{0}, \ldots, 10^{3} \mathrm{~Hz}^{16,17,22}$ In order to replenish the dissociated molecules inside the continuously irradiated area by gas transport only, we need $\widetilde{\tau} \rightarrow 1$, i.e., the precursor supply frequency $s J / n_{0}$ should exceed $\sigma f_{0}$, being equivalent to $>2 \times 10^{3} \mathrm{ML} / \mathrm{s}$ (monolayers per second) for FEB. This corresponds to a precursor flux on the substrate of $J=2$ $\times 10^{17}$ molecules $\mathrm{cm}^{-2} \mathrm{~s}^{-1}$, setting $s=1$ and taking $n_{0}$ $=10^{14} \mathrm{~cm}^{-2}$ as typical value. For FIB several orders of magnitude larger gas supply would be needed. We would like to point out that a precursor supply of $10^{6} \mathrm{ML} / \mathrm{s}$ is equivalent to a local pressure of roughly 1 mbar. Depending on the volume of increased local pressure above the substrate surface, this can lead to considerable molecule ionization by collisions with charged particles. Gas phase migration of ionized molecules is well known from the charge compensation effect in environmental SEMs. Although this sets a limit to our present model these conditions are rarely reached. In contrary, above estimations clearly suggest that most of the FEB and FIB processing experiments were performed in the precursor-limited regime limiting the minimum deposit or etch size.

In the following we show under which conditions substantial replenishment by surface diffusion can be expected. Solving Eq. (2) numerically for steady-state $(d n / d t=0)$, the boundary conditions $n(r \rightarrow \infty)=n_{\text {out }}=s J \tau_{\text {out }}$ and $d n(r=0) / d r$ $=0$, and with a Gaussian distribution $f(r)=f_{0} \exp \left(-r^{2} / 2 a^{2}\right)$, we get $n(r)$ and finally $R(r)$. Figure 2 shows a plot of $R(r)$ against the dimensionless variable $\tilde{r}=2 r / \mathrm{FWHM}_{B}$ for a given depletion. Diffusive replenishment is described by the dimensionless ratio $\widetilde{\rho}=2 \rho_{\text {in }} / \mathrm{FWHM}_{B}$, relating the molecule diffusion path in the center of the beam $\rho_{\text {in }}=\left(D \tau_{\text {in }}\right)^{1 / 2}, D$ being the diffusion coefficient, to the beam size. For $\tilde{\rho}=0$, the deposit or etch shape is defined by Eq. (3) and its resolution by Eq. (4). With increasing diffusive replenishment

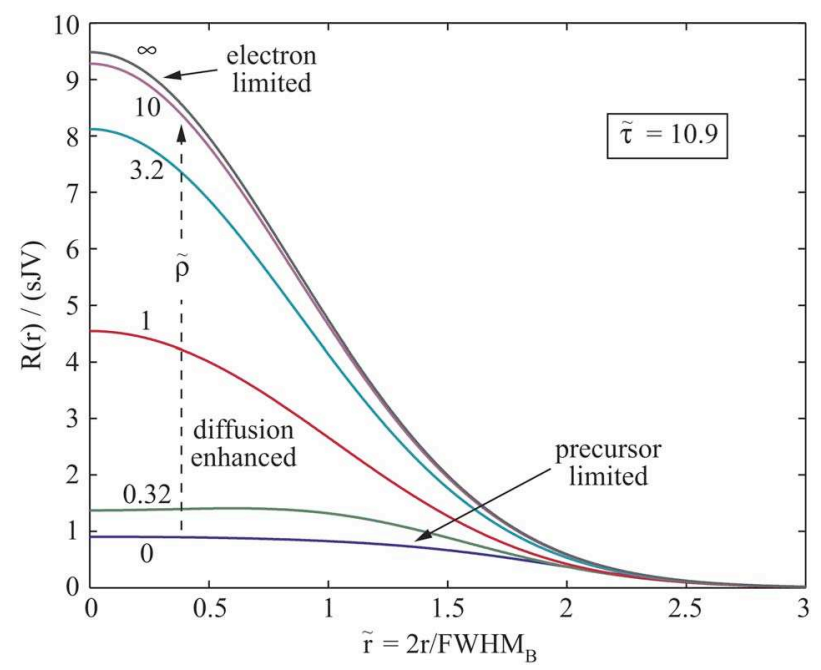

FIG. 2. Normalized steady-state deposition rate at indicated depletion from Eq. (1) representing the deposit shape. Diffusive replenishment $\widetilde{\rho}$ $=2 \rho_{\text {in }} / \mathrm{FWHM}_{B}$ is varied. Note the shape transition from flat top, $\tilde{\rho}=0$, indented, $\widetilde{\rho}=0.32$, to round, $\widetilde{\rho}=1$, to Gaussian, $\widetilde{\rho}=\infty$.

deposits change from flat-top shape to indented and round shapes, since adsorbed molecules increasingly reach the center of the irradiated area before being dissociated. Both deposition rate and resolution increase. The maximum diffusion enhancement in deposition rate becomes $R(\widetilde{\rho}=\infty) / R(\widetilde{\rho}=0)$ $=\tilde{\tau}$ at $r=0$. For $\tilde{\rho} \rightarrow \infty$, Eq. (3) simplifies to $R(r)$ $=s J \tau_{\text {out }} V \sigma f(r)$ since any depletion is entirely compensated by diffusion and a permanent monolayer coverage provided. In other words, the electron-limited regime is established and the deposit shape corresponds to the electron beam distribution $f(r)$.

Figure 3 represents a graph relating the dimensionless resolution to irradiative depletion and diffusive replenishment. Evidently, when the diffusion path inside the irradiated area becomes at least comparable to the size of the electron beam distribution $\widetilde{\rho} \geq 2$, the resolution is $\widetilde{\varphi} \leq 1.03$ for any

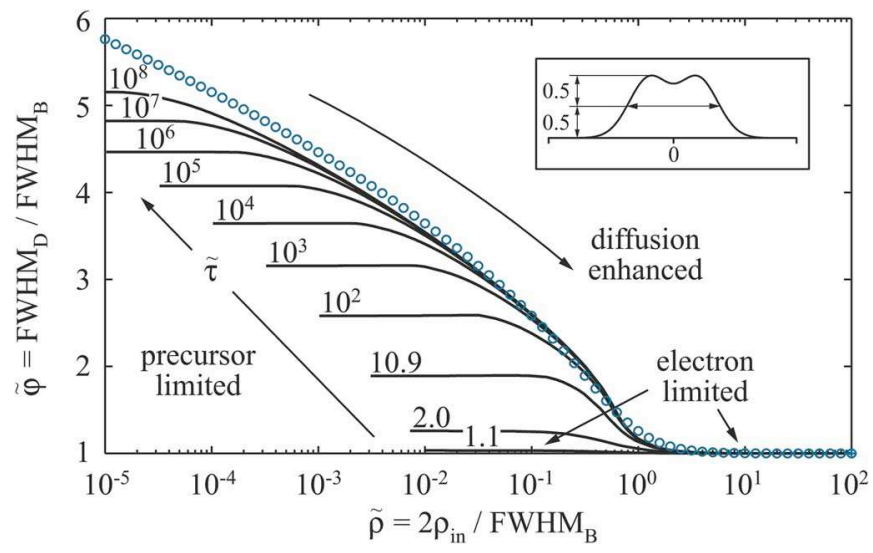

FIG. 3. Normalized deposit size vs normalized diffusion path for varying depletion (indicated) for a Gaussian distribution $f(r)$. At $\widetilde{\rho}=2$ the diffusion path equals the beam size. Curve for $\tilde{\tau}=10.9$ corresponds to the FWHMs of shapes in Fig. 2. Circles represent Eq. (5). Inset shows the $\mathrm{FWHM}_{D}$ definition of indented deposits. 


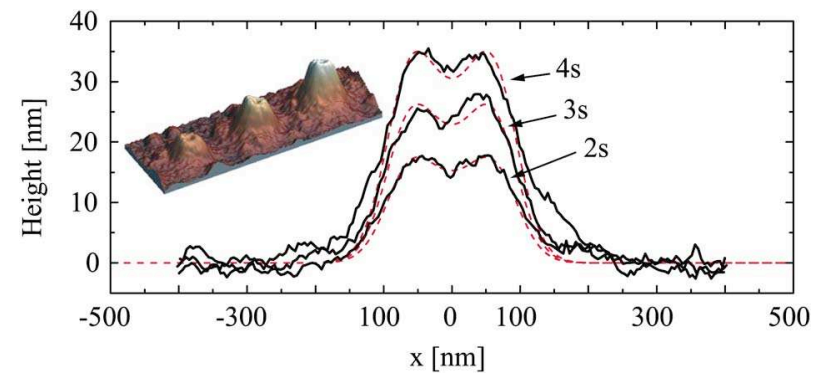

FIG. 4. AFM image and line scans of FEB deposits from $\mathrm{Cu}(\mathrm{hfac})_{2}$ precursor. Exposure times are indicated. Indented apex shapes are due to depletion. Dashed lines represent fits of Eq. (1) with $\sigma=0.6 \mathrm{~nm}^{2}$ (=molecule size), $\tau=10^{-3} \mathrm{~s}$ (typical value), and $D=4 \times 10^{-7} \mathrm{~cm}^{2} \mathrm{~s}^{-1}$. Other deposition parameters: Gaussian beam of $\mathrm{FWHM}_{B}=110 \mathrm{~nm} \quad(5 \mathrm{keV}), f_{0}=9$ $\times 10^{4} \mathrm{~nm}^{-2} \mathrm{~s}^{-1}, s J / n_{0}=10 \mathrm{ML} / \mathrm{s}$.

depletion, i.e., the deposit size is within $3 \%$ close to $\mathrm{FWHM}_{B}$. The deposits become broader than the electron beam for $\tilde{\tau}>1$ and small $\tilde{\rho}$, branching out into constant maximum size given by Eq. (4) at negligible diffusive replenishment $\widetilde{\rho} \rightarrow 0$. Figure 3 holds independently of how diffusive replenishment is experimentally achieved: either via the beam size $\mathrm{FWHM}_{B}$ (using the focus of the beam) or via the diffusion path $\rho_{\text {in }}$ (changing precursor diffusion). The second scaling law of resolution as a function of diffusive replenishment $\widetilde{\varphi}(\widetilde{\rho})$ is obtained as (see the circles in Fig. 3)

$$
\widetilde{\varphi} \approx\left\{\log _{2}\left(2+\tilde{\rho}^{-2}\right)\right\}^{1 / 2} .
$$

The smaller value of Eqs. (4) and (5) would define the minimum deposit (or etch) size with respect to the beam size. Since $\widetilde{\varphi}$ is easy to determine experimentally, the two scaling laws allow for rapid estimations of irradiative depletion and diffusive replenishment.

\section{EXPERIMENTAL}

Figure 3 presents shape measurements of FEB deposits obtained with a SEM compatible atomic force microscope (AFM). The advantage is that AFM reveals indented shapes which are difficult to resolve in SEMs due to edge contrast effects. We used $\mathrm{Cu}(\mathrm{II})$-hexafluoroacetylacetonate precursor molecules impinging on a native Silicon substrate with a local flux of $J / n_{0}=10 \mathrm{ML} / \mathrm{s}$ and irradiated with a $5 \mathrm{keV}$ Gaussian electron beam $\left(f_{0}=9 \times 10^{4} \mathrm{~nm}^{-2} \mathrm{~s}^{-1}\right.$ and $\left.\mathrm{FWHM}_{B}=110 \mathrm{~nm}\right)$. An indented shape with $\mathrm{FWHM}_{D}$ $=200 \mathrm{~nm}$ is observed giving $\tilde{\varphi}=1.8$. Equation (4) yields $\tilde{\tau}$ $=8.9$. Assuming as typical values $\tau=10^{-3} \mathrm{~s}$ and $s=1$ results in $\sigma \sim 0.09 \mathrm{~nm}^{2}$. From Eq. (5) follows $\tilde{\rho}=0.37$, i.e., $\rho_{\text {in }}$ $=20 \mathrm{~nm}$. Using the relation $\rho_{\text {in }} \simeq\left(D / \sigma f_{0}\right)^{1 / 2}$ for $\tilde{\tau} \gg 1$ results in $D \sim 3 \times 10^{-8} \mathrm{~cm}^{2} \mathrm{~s}^{-1}$.

The above values for the cross section and diffusion coefficient represent lower limit estimations since the same resolution $\tilde{\varphi}$ can be obtained with larger depletion and larger diffusive replenishment; see Fig. 3. Upper-limit estimates can be obtained by taking as maximum dissociation cross section the molecule size $\sigma=0.6 \mathrm{~nm}^{2} .^{25}$ This gives $\tilde{\tau}=60$. The corresponding diffusion coefficient is derived from the shape fit in Fig. 4 to be $D=4 \times 10^{-7} \mathrm{~cm}^{2} \mathrm{~s}^{-1}$. Hence, with one experiment the ranges of the parameters $D$ and $\sigma$ can be estimated.

Finally, we estimate diffusion coefficients and exposure times needed for establishing the electron-limited regime under typical irradiation conditions summarized in Table I. Compensation of depletion by surface diffusion requires a molecule diffusion path at least comparable to the beam size, $\tilde{\rho} \geq 2$; see Fig. 3. Together with the relation $\rho_{\text {in }} \simeq\left(D / \sigma f_{0}\right)^{1 / 2}$ for $\tilde{\tau} \gg 1$ we obtain $D=10^{-10} \ldots 10^{-3} \mathrm{~cm}^{2} \mathrm{~s}^{-1}$ for FEB and $D=10^{-6} \ldots 10^{-2} \mathrm{~cm}^{2} \mathrm{~s}^{-1}$ for FIB. When the condition $\tilde{\rho} \geq 2$ cannot be met in a continuous exposure experiment, pulsed beams can be employed. Basically, the short exposure and long refresh times prevent irradiative depletion. The refresh times are in the millisecond range. ${ }^{22,23}$ The exposure time scales can be estimated by solving Eq. (2) without diffusion term for $n(t)$. An exponential decrease of the effective residence time at the center of the irradiated area is obtained, $\tau_{\text {in }}(t) \sim \tau_{\text {out }} \exp (-k t)$, where $k=1 / \tau_{\text {in }} \simeq \sigma f_{0}$ for large dissociation frequencies. Inserting into Eq. (4) yields the third scaling law of resolution as function of exposure time $\tilde{\varphi}(t)$, for $t \leqslant k^{-1} \ln (\widetilde{\tau})$,

$$
\widetilde{\varphi}=\left\{\log _{2}(1+\exp (k t)\}^{1 / 2} .\right.
$$

Keeping the deposit size $10 \%$ close to the beam size, $\widetilde{\varphi}$ $=1.1$, gives $t \leq 0.27\left(\sigma f_{0}\right)^{-1}$ according to Eq. (6). This translates for FEB into $t_{\mathrm{FEB}} \leq 30 \mathrm{~ns} \ldots 0.3 \mathrm{~ms}$ and for FIB into $t_{\mathrm{FIB}} \leq 1$ ns...0.1 $\mu$ s using $\sigma f_{0}$ values from Table I. The low end of these time scales has not yet been explored in focused particle beam induced processing. For the peak function $f(r)=f_{0} \exp (-a r)$ roughly describing the emitted secondary electron distribution, the outer exponent in Eqs. (4)-(6) becomes 1.

\section{CONCLUSIONS}

In conclusion, we derived three scaling laws quantifying the crucial role of irradiative depletion and diffusive replenishment on deposit and etch resolution for two distributions: a Gaussian and an exponential decay. Our model is applicable to gas-assisted deposition and etching with focused electron- and ion beams where precursor shadow effects, forward scattering, or sidewall secondary electron emission are negligible. We demonstrated how physical parameters can be estimated from fitting experimental deposit shapes with our model. An extension of the studies to different beam shapes, pulsed beams, physical sputtering, and temperatures is straightforward. It will enable the systematic determination of all physical key parameters involved in the process, thus opening the door to the controlled fabrication of tailored nanoscale devices by charged particle beam induced CVD and etching.

\section{ACKNOWLEDGMENTS}

We acknowledge financial support from the European Commission, FP6 Integrated Project NanoHand (IST-5034274).

${ }^{1}$ C. V. Oatley, J. Appl. Phys. 53, R1 (1982). 
${ }^{2}$ M. F. Yu, O. Lourie, M. J. Dyer, K. Moloni, T. F. Kelly, and R. S. Ruoff, Science 287, 637 (2000).

${ }^{3}$ I. Utke, P. Hoffmann, R. Berger, and L. Scandella, Appl. Phys. Lett. 80, 4792 (2002).

${ }^{4}$ K. Edinger, T. Gotszalk, and I. Rangelow, J. Vac. Sci. Technol. B 19, 2856 (2001).

${ }^{5}$ M. Castagne, M. Benfedda, S. Lahimer, P. Falgayrettes, and J. P. Fillard, Ultramicroscopy 76, 187 (1999).

${ }^{6}$ E. J. Sanchez, J. T. Krug, and X. S. Xie, Rev. Sci. Instrum. 73, 3901 (2002).

${ }^{7}$ T. Nagata, P. Ahmet, Y. Sakuma, T. Sekiguchi, and T. Chikyow, Appl. Phys. Lett. 87, 013103 (2005).

${ }^{8}$ V. Gopal, V. R. Radmilovic, C. Daraio, S. Jin, P. D. Yang, and E. A. Stach, Nano Lett. 4, 2059 (2004).

${ }^{9}$ J. Nilsson, J. R. I. Lee, T. V. Ratto, and S. E. Letant, Adv. Mater. 18, 427 (2006).

${ }^{10}$ C. Danelon, C. Santschi, J. Brugger, and H. Vogel, Langmuir 22, 10711 (2006).

${ }^{11}$ T. E. Allen, R. R. Kunz, and T. M. Mayer, J. Vac. Sci. Technol. B 6, 2057 (1988).
${ }^{12}$ A. D. Dubner and A. Wagner, J. Vac. Sci. Technol. B 7, 1950 (1989).

${ }^{13}$ N. Silvis-Cividjian, C. W. Hagen, and P. Kruit, J. Appl. Phys. 98, 084905 (2005).

${ }^{14}$ C. W. Hagen, N. Silvis-Cividjian, and P. Kruit, Scanning 28, 204 (2006).

${ }^{15}$ K. H. Muller, Optik (Stuttgart) 33, 296 (1971).

${ }^{16}$ S. Haraichi and M. Komuro, Jpn. J. Appl. Phys., Part 1 32, 6168 (1993).

${ }^{17}$ V. Scheuer, H. Koops, and T. Tschudi, Microelectron. Eng. 5, 423 (1986).

${ }^{18}$ K. T. Kohlmannvonplaten, L. M. Buchmann, H. C. Petzold, and W. H. Brunger, J. Vac. Sci. Technol. B 10, 2690 (1992).

${ }^{19}$ D. Kunze, O. Peters, and G. Sauerbrey, Z. Angew. Phys. 22, 69 (1967).

${ }^{20}$ H. C. Petzold and P. J. Heard, J. Vac. Sci. Technol. B 9, 2664 (1991).

${ }^{21}$ P. G. Blauner, J. S. Ro, Y. Butt, and J. Melngailis, J. Vac. Sci. Technol. B 7, 609 (1989).

${ }^{22}$ S. Lipp, L. Frey, C. Lehrer, B. Frank, E. Demm, S. Pauthner, and H. Ryssel, J. Vac. Sci. Technol. B 14, 3920 (1996).

${ }^{23}$ K. Edinger and T. Kraus, J. Vac. Sci. Technol. B 18, 3190 (2000).

${ }^{24}$ J. S. Ro, C. V. Thompson, and J. Melngailis, J. Vac. Sci. Technol. B 12, 73 (1994).

${ }^{25}$ S. A. Gromilov, I. A. Baidina, P. A. Stabnikov, and G. V. Romanenko, J. Struct. Chem. 45, 476 (2004). 